\title{
Hiperglucemia preoperatoria en pacientes no diabéticos sometidos a cirugías electivas
}

\author{
Preoperative hyperglycemia in patients without diabetes \\ mellitus subjected to elective surgeries
}

\author{
Xiaojin Zhou, ${ }^{*}$ José Manuel Portela Ortiz, ${ }^{\ddagger}$ Guadalupe Zaragoza Lemus, ${ }^{\S}$ \\ Delia Brenda Paola Ocampo Valencia*
}

Citar como: Zhou X, Portela OJM, Zaragoza LG, Ocampo VDBP. Hiperglucemia preoperatoria en pacientes no diabéticos sometidos a cirugías electivas. Acta Med GA. 2021; 19 (4): 506-509. https://dx.doi.org/10.35366/102536

\section{Resumen}

Introducción: La hiperglucemia aumenta la morbimortalidad perioperatoria. Por lo tanto su reconocimiento oportuno favorece un mejor resultado quirúrgico. Los pacientes con hiperglucemia tienen más probabilidades de ser hospitalizados que aquéllos sin diabetes porque muchos de ellos no están diagnosticados antes de su hospitalización. Objetivo: Determinar la incidencia de hiperglicemia preoperatoria en pacientes sin diagnóstico de diabetes mellitus. Material y métodos: Se incluyeron pacientes con ASA 1 y 2 , edad > 18 años, cirugía electiva de forma no aleatorizada. Estudio observacional, prospectivo, transversal. Se registró la glicemia capilar preoperatoria. El análisis estadístico fue realizado mediante sumatorias simples. Resultados: Se obtuvo un total de 509 pacientes con ASA 1 y 2, de los cuales se excluyeron 116 pacientes $(22.7 \%)$. Se obtuvo una muestra final de 393 . Se identificaron 106 pacientes con hiperglucemia preoperatoria (27\%), de los cuáles se encontraron ocho pacientes (7.54\%) con glicemia $\geq 126 \mathrm{mg} / \mathrm{dL}$. Se subdividieron los pacientes por grupos de edad, donde se observó que la incidencia de hiperglucemia aumenta con la edad, la media de la hiperglucemia capilar fue de $94.58 \mathrm{mg} / \mathrm{dL} \pm 14.24$. Conclusiones: En este trabajo la hiperglucemia se presentó $17.7 \%$ en pacientes de $18-40$ años y hasta $62 \%$ en pacientes mayores de 80 años sin DM. La determinación de glicemia debe considerarse como un examen preoperatorio eficiente.

Palabras clave: Hiperglucemia preoperatoria, diabetes mellitus, cirugía electiva.

\section{Abstract}

Introduction: Hyperglycemia can increase perioperative morbidity and mortality. Therefore, its timely recognition favors a better surgical result. Patients with hyperglycemia are more likely to be hospitalized than those without diabetes, as many of these patients are not diagnosed prior to hospitalization. Objective: To determine the incidence of preoperative hyperglycemia in patients who do not have a diagnosis of diabetes mellitus. Material and methods: Patients with ASA 1 and 2, age $>18$ years, elective surgery were included. An observational, prospective, cross-sectional study was carried out. Preoperative capillary glycemia was recorded. Data collection is expressed in averages and percentages. Results: 509 patients with ASA 1 and 2 of which 116 patients (22.7\%) were excluded. Patients included $393(n=393) .106$ patients with preoperative hyperglycemia $(27 \%)$ were identified and eight patients (7.54\%) with glycemia $\geq 126 \mathrm{mg} / \mathrm{dL}$ were found, mean hyperglycemia was $94 \mathrm{mg} / \mathrm{dL} \pm 14.2$. Is subdivided hyperglycemic patients by age group, and the incidence of hyperglycemia increases with age. Conclusions: In this study, hyperglycemia was present in $17.7 \%$ of patients of $18-40$ years and up to $62 \%$ in patients more than 80 years without DM. The determination of glucose must be considered as an efficient preoperative test.

Keywords: Preoperative hyperglycemia, diabetes mellitus, elective surgery.
* Residente de la Especialidad en Anestesiología.

‡ Jefe del Servicio de Anestesiología, Profesor titular del Curso de Anestesiología.

$\S$ Profesora auxiliar del Curso de Anestesiología. Instituto Nacional de Rehabilitación.

Facultad Mexicana de Medicina. Universidad La Salle México.
Correspondencia:

Dra. Xiaojin Zhou

Correo electrónico: zhouxiaojin91@gmail.com

Aceptado: 01-03-2021.

www.medigraphic.com/actamedica

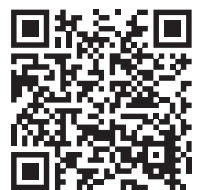




\section{INTRODUCCIÓN}

La hiperglucemia es un desorden metabólico de múltiples etiologías que afecta la mayoría de los órganos y sistemas. Esta condición puede incrementar la morbilidad y la mortalidad perioperatoria. ${ }^{1}$ Además se asocia a resultados adversos como mayor incidencia de infecciones nosocomiales como de las heridas quirúrgicas, catéteres, de la vía urinaria y de la vía respiratoria; también cursa con mayor incidencia de eventos trombóticos y cardiovasculares, y mayor estancia intrahospitalaria; por lo tanto, su reconocimiento y manejo oportuno favorece un mejor resultado quirúrgico. ${ }^{2}$ Los resultados de la encuesta Nacional de Salud reportan que la prevalencia total de diabetes en México es de $13.7 \%$ (9.5\% diagnosticada y $4.1 \%$ no diagnosticada). ${ }^{3}$ La hiperglucemia se define como una concentración de glucosa en ayuno $\geq 100 \mathrm{mg} / \mathrm{dL}$, pero por debajo del valor necesario para diagnosticar diabetes mellitus $(<126 \mathrm{mg} / \mathrm{dL}$ se considera simple resistencia a la insulina). ${ }^{4}$ Los pacientes con hiperglucemia tienen más probabilidades de ser hospitalizados que aquéllos sin diabetes, siendo que muchos de estos pacientes no están diagnosticados antes de su hospitalización. ${ }^{5}$ La meta es mantener niveles razonables de glucemia en el perioperatorio entre 110-180 mg/dL, que eviten las alteraciones metabólicas e hidroelectrolíticas agudas, lo cual requiere de una adecuada monitorización de la glucemia durante todo el procedimiento. Los niveles de glucosa elevados en el preoperatorio ( $>200 \mathrm{mg} / \mathrm{dL}$ ) se asocian a infección de herida quirúrgica debido a que deterioran la función de los neutrófilos y provocan una sobreproducción de especies reactivas de oxígeno, ácidos libres y mediadores inflamatorios, estos cambios fisiopatológicos contribuyen al daño celular directo y las disfunciones vasculares e inmunes. ${ }^{6-8}$ Por lo tanto, el objetivo de este estudio fue valorar qué porcentaje de pacientes no diabéticos presentan un estado hiperglucémico preoperatorio.

\section{MATERIAL Y MÉTODOS}

Previa autorización por los comités de investigación y ética en investigación de salud institucionales se realizó un estudio observacional, prospectivo, transversal, donde se incluyeron pacientes sometidos a cirugía electiva en el Hospital Angeles Pedregal. Se valoró a los pacientes en el área preoperatoria y se registró su nivel de glucemia del reporte de laboratorio para incluirse al estudio, la toma debía ser del día de la cirugía, si el reporte era de días previos se tomaba muestra de glucosa capilar con un equipo (de marca FreeStyle Optium NeoH con tiras reactivas Abbott), los pacientes que se incluyeron en el estudio fueron de cirugía electiva, con valoración de estado físico de la American Society of Anesthesiologist (ASA 1 y 2), con una edad mayor de 18 años y se excluyeron pacientes diabéticos, o que tuvieran patologías que produjeran alteración del metabolismo de la glucosa, uso crónico de esteroides, pacientes embarazadas, pacientes con VIH-SIDA, o con uso de eritropoyetina.

Análisis estadístico. Todos los datos se sometieron a cálculos de pruebas estadísticas de promedios, porcentajes simples y sumatorias en Excel V2013.

\section{RESULTADOS}

En el periodo de estudio fue de junio a julio 2019, se incluyó un total de 393 pacientes, la media de peso fue $71.59 \mathrm{Kg} \pm 14.3$, media de talla $1.66 \mathrm{~m} \pm 0.09$, la media de IMC fue 25.77, media de edad fue 47.83 años \pm 17.17 y la media de glicemia capilar $94.58 \mathrm{mg} / \mathrm{dL} \pm 14.24$ (Tabla 1). De los cuales, el estado físico ASA 1 que correspondió a $50.13 \%$ y ASA 2 con $49.87 \%$ se subdividieron en cuatro subgrupos de edad, $52.93 \%$ fueron del sexo femenino y $47.07 \%$ masculino (Tabla 2). Se identificaron en total 106 pacientes $(27 \%)$ con hiperglucemia preoperatoria, de los cuales 98 (92.5\%) presentaban una glucemia entre $101 \mathrm{y}$ $125 \mathrm{mg} / \mathrm{dL}$, y ocho pacientes (7.5\%) tenían glicemia $\geq 126$ $\mathrm{mg} / \mathrm{dL}$. El grupo de edad que mayor incidencia de pacientes con hiperglucemia mostró fue el de mayores de 80 años (Tabla 3). De los pacientes con hiperglucemia, ninguno tuvo complicaciones agudas durante el transoperatorio, dos pacientes presentaron infección postoperatoria en herida quirúrgica.

\section{DISCUSIÓN}

La hiperglicemia preoperatoria es multifactorial, se deben realizar estudios complementarios para determinar su causa. Es un factor de riesgo de desarrollar diabetes mellitus, por lo que se recomienda la determinación de hemoglobina glicosilada dentro de los estudios preoperatorios. . $^{5,6}$

La hiperglucemia perioperatoria en Estados Unidos es de $20-40 \%$ en pacientes que son sometidos a cirugías y

\begin{tabular}{|lcc|}
\hline \multicolumn{2}{c}{ Tabla 1: Descripción demográfica } \\
de la muestra. N = 393.
\end{tabular}


Tabla 2: Descripción de la muestra por American Society of Anesthesiologist. $\mathrm{N}=393$.

\begin{tabular}{ccc} 
Años & $\begin{array}{c}\text { ASA 1 (197*) } \\
n(\%)\end{array}$ & $\begin{array}{c}\text { ASA 2 (196*) } \\
n(\%)\end{array}$ \\
\hline $18-40$ & $99(50)$ & $39(20)$ \\
$41-60$ & $77(39)$ & $74(38)$ \\
$61-80$ & $21(11)$ & $75(38)$ \\
$>80$ & $0(0)$ & $8(4)$ \\
\hline
\end{tabular}

$\mathrm{ASA}=$ American Society of Anesthesiologist,${ }^{*}=$ pacientes.

Tabla 3: Pacientes con hiperglucemia por grupo de edad. $\mathrm{N}=393$.

\begin{tabular}{|ccc|}
\hline Años & Hiperglucemia & $\%$ \\
\hline $18-40$ & $25 / 141$ & 17.7 \\
$41-60$ & $41 / 151$ & 27.0 \\
$61-80$ & $35 / 96$ & 36.0 \\
$>80$ & $5 / 8$ & 62.0 \\
\hline
\end{tabular}

aproximadamente $80 \%$ después de cirugías cardiacas. De 12 a $30 \%$ de los pacientes que tienen hiperglucemia intraoperatoria o postoperatoria no se saben diabéticos antes de la cirugía, un estado que a menudo se describe como "hiperglucemia por estrés". Además, $60 \%$ de los pacientes ingresados con nueva hiperglucemia tuvieron diabetes confirmada un año después. Los estudios observacionales realizados en Estados Unidos reportan que la hiperglucemia está presente en 32-38\% de los pacientes hospitalizados que no están en una unidad de cuidados intensivos ( $\mathrm{UCl}$ ) y de ellos, aproximadamente una tercera parte no tienen diagnóstico previo de diabetes. Los pacientes quirúrgicos que desarrollan hiperglucemia muestran un incremento en el riesgo de resultados adversos, mayor índice de infecciones principalmente de la herida quirúrgica. Por esta razón, la valoración preoperatoria es fundamental. ${ }^{7,8}$ La incidencia de mortalidad a un año es de 3-5\% en pacientes con normoglucemia, versus $12 \%$ en pacientes con hiperglucemia $>216 \mathrm{mg} / \mathrm{dL}$.

Es muy importante el manejo perioperatorio para el control de la glucemia, el objetivo es mantener entre 110-180 mg/dL (6.1-10). La importancia del control glucémico de los pacientes hiperglucémicos se debe a que los procedimientos quirúrgicos pueden dar como resultado diferentes cambios metabólicos que pueden alterar la homeostasis de la glucosa con la consecuente hiperglucemia, la cual es un factor de riesgo de desarrollar sepsis en el postoperatorio, disfunción endotelial, isquemia cerebral y alteración del proceso de cicatrización de las heridas. ${ }^{9-11}$

Por lo tanto, el control metabólico es pieza clave en la conducta perioperatoria en estos pacientes, la participación multidisciplinaria (médico internista, cirujano y anestesiólogo) definirá de manera conjunta las estrategias quirúrgicas y anestésicas óptimas para un mejor desenlace durante y después de la cirugía. ${ }^{12}$ Sin embargo, ningún estudio prospectivo aleatorizado ha determinado la importancia de control preoperatorio y el resultado clínico. En 2014, la FDA (food and drug administration) emitió un borrador de orientación que describe que 99\% de las lecturas de prueba capilar > $70 \mathrm{mg} / \mathrm{dL}$ se encuentra dentro de $10 \%$ de los valores de referencia del laboratorio central y que todas las lecturas de glucosa de sangre $<70 \mathrm{mg} / \mathrm{dL}$ está dentro de los $7 \mathrm{mg} / \mathrm{dL}^{13}$

Desafortunadamente, el tamaño pequeño de la muestra limita la capacidad de poder llegar a conclusiones contundentes y definitivas así como extrapolar los resultados y considerarlos en otras poblaciones. Se necesita un estudio más amplio que incluya mayor número de pacientes con un seguimiento exhaustivo del índice de infecciones en el posoperatorio.

\section{CONCLUSIÓN}

La incidencia de hiperglucemia preoperatoria en pacientes sin diagnóstico de diabetes mellitus sometidos a cirugía electiva fue aumentando con la edad hasta en $62 \%$ en pacientes mayores de 80 años. Por lo que se concluye que el diagnóstico de hiperglucemia debe siempre buscarse en los pacientes quirúrgicos de cualquier edad, pero sobre todo de edades avanzadas por su alto impacto en el resultado clínico adverso y su alta prevalencia. Consideramos que realizar una glicemia capilar es un método eficiente para detectar el grupo de pacientes de alto riesgo.

\section{REFERENCIAS}

1. Clement S, Braithwaite SS, Magee MF, Ahmann A, Smith EP, Schafer RG et al. Management of diabetes and hyperglycemia in hospitals. Diabetes Care. 2004; 27 (2): 553-591.

2. Smiley DD, Umpierrez GE. Perioperative glucose control in the diabetic or nondiabetic patient. South Med J. 2006; 99 (6): 580-591.

3. http://www.insp.mx/avisos/3652-diabetes-en-mexico.html

4. Alberti KGMM, Gill GV. The care of the diabetic patient during surgery. En: Alberti KGMM, Zimmet P, DeFronzo RA, Keen H, eds. International textbook of diabetes mellitus. 2nd ed. Chichester: Wiley; 1997, 1243-1253.

5. Debing E, Aerden D, Van den Brande P. Diabetes mellitus is a predictor for early adverse outcome after carotid endarterectomy. Vasc Endovascular Surg. 2011; 45 (1): 28-32. 
6. Gandhi GY, Nuttall GA, Abel MD, Mullany CJ, Schaff HV, O'Brien $P C$ et al. Intensive intraoperative insulin therapy versus conventional glucose management during cardiac surgery: a randomized trial. Ann Intern Med. 2007; 20: 233-243.

7. Chan RP, Galas FR, Hajjar LA, Bello CN, Piccioni MA, Auler JO Jr. Intensive perioperative glucose control does not improve outcomes of patients submitted to open-heart surgery: a randomized controlled trial. Clinics (Sao Paulo). 2009; 64 (1): 51-60.

8. Leibowitz G, Raizman E, Brezis M, Glaser B, Raz I, Shapira O. Effects of moderate intensity glycemic control after cardiac surgery. Ann Thorac Surg. 2010; 90 (6): 1825-1832.

9. The NICE-SUGAR Study Investigators. Intensive verses conventional glucose control in critically ill patients. N Engl J Med. 2009; 360: 1283-1297.
10. Pontes JPJ, Mendes FF, Vasconcelos MM, Batista NR. Evaluation and perioperative management of patients with diabetes mellitus. Braz J Anesthesiol. 2018; 68 (1): 75-86.

11. Alserius T, Anderson RE, Hammar N, Nordqvist T, Ivert T. Elevated glycosylated haemoglobin (HbA1c) is a risk marker in coronary artery bypass surgery. Scand Cardiovasc J. 2008; 42 (6): 392-398.

12. Knapik P, Ciesla D, Filipiak K, Knapik M, Zembala M. Prevalence and clinical significance of elevated preoperative glycosylated hemoglobin in diabetic patients scheduled for coronary artery surgery. Eur J Cardiothorac Surg. 2011; 39 (4): 484-489.

13. Duggan EW, Carlson K, Umpierrez GE. Perioperative hyperglycemia management: an update [published correction appears in anesthesiology. 2018; 129 (5): 1053. Anesthesiology. 2017; 126 (3): 547-560. 